\title{
Measurement of spinal cord area in clinically isolated syndromes suggestive of multiple sclerosis
}

\author{
P A Brex, S M Leary, J I O’Riordan, K A Miszkiel, G T Plant, A J Thompson, D H Miller
}

\begin{abstract}
Atrophy of the spinal cord is known to occur in multiple sclerosis but the cause and the timing of its onset are not clear. Recent evidence suggests that atrophy may start to occur early in the disease. The aim was to determine whether atrophy of the spinal cord could be detected in vivo using MRI techniques, in patients presenting with a clinically isolated syndrome, which in many cases is the earliest clinical stage of multiple sclerosis.
\end{abstract}

The cross sectional area of the spinal cord was measured in 43 patients presenting with a clinically isolated syndrome and 15 matched controls. $T 2$ weighted imaging of the brain was also performed to determine the number and volume of high signal lesions consistent with disseminated demyelination. Both patients and controls were restudied after 1 year.

The spinal cord area was significantly smaller in the $74 \%$ of patients with an abnormal brain MRI at presentation than in controls (mean areas $73.9 \mathrm{~mm}^{2}$ and 78.1 $\mathrm{mm}^{2}$ respectively, $\mathrm{p}=0.03$ ). No significant difference was found in the spinal cord area between controls and patients with normal baseline brain imaging. The annual rate of change in patients did not differ significantly from controls.

In conclusion, the finding of a smaller cord area in the subgroup of patients with clinically isolated syndrome with the highest risk of developing multiple sclerosisthat is, with an abnormal brain MRI, suggests that atrophy has developed in some patients with multiple sclerosis even before their first clinical symptoms. However, the lack of a detectable change in cord area over 1 year of follow up contrasts strikingly with the results of an earlier study of patients with relapsingremitting multiple sclerosis, suggesting that the rate of atrophy increases as the disease becomes more established.

(F Neurol Neurosurg Psychiatry 2001;70:544-547)

Keywords: clinically isolated syndromes; spinal cord atrophy; multiple sclerosis

Correspondence to: Professor DH Miller d.miller@ion.ucl.ac.uk

Received 15 May 2000 and in revised form

19 October 2000

Accepted 26 October 2000
Atrophy of the brain and spinal cord are common postmortem findings in patients with chronic multiple sclerosis. The cause and the stage of the disease when atrophy starts to occur, however, are not known. Magnetic resonance imaging provides a method for examining the development of atrophy in vivo and has demonstrated that atrophy of the spinal cord, a site commonly involved in multiple sclerosis, correlates highly with disability. ${ }^{1-3}$ This suggests that atrophy reflects pathological processes which are of functional importance. Although demyelination may contribute to atrophy, it would seem insufficient to explain the correlation with progressive neurological deterioration and it is more likely that axonal loss, which is also known to occur in multiple sclerosis, is the predominant cause, particularly in the later stages of the disease. The positive correlation between cerebral atrophy and white matter $\mathrm{N}$-acetyl aspartate concentration (a neuronal marker) in secondary progressive multiple sclerosis suggests that this is indeed the case. ${ }^{4}$ Histopathological reports have found axonal damage in early inflammatory lesions ${ }^{5}$ suggesting that axonal loss may be present even in the early stages of the disease. A recent postmortem study of the spinal cord has confirmed this, finding a loss of axons in the spinal cord in some patients with multiple sclerosis with a short disease duration. ${ }^{6}$

Stevenson et al, using a highly reproducible method, which measured the cross sectional spinal cord area at the C2 level, showed that the annual rate of cord atrophy in patients with relapsing-remitting multiple sclerosis was significantly greater than in matched controls. ${ }^{2}$ This study aimed to determine whether or not, using the same technique, spinal cord atrophy could be detected in patients after a clinically isolated syndrome, which for many is the earliest clinical stage of multiple sclerosis. ${ }^{7}$

\section{Methods}

SUBJECTS

Forty three patients from a prospective, longitudinal multisequence MRI study of clinically isolated syndromes were investigated. A clinically isolated syndrome was defined by the occurrence of a presumed inflammatory demyelinating event of acute onset in any part of the CNS in a person without a history of neurological symptoms suggestive of demyelination. In all patients investigations were carried out as necessary to exclude alternative diagnoses. The study had been approved by local medical ethics committees. Informed 
consent was obtained from all patients before entry. Fifteen age and sex matched healthy controls were also studied.

\section{MRI SEQUENCES}

All MR studies took place on a 1.5 Tesla Signa Horizon EchoSpeed scanner (General Electric, Milwaukee, Wisconsin, USA). Imaging was performed as soon as practicable after the presenting symptom onset and at any rate within 3 months (baseline) and again after about 1 year. As part of the multisequence MRI study, an intravenous bolus of gadolinium-DTPA (0.1 $\mathrm{mmol} / \mathrm{kg}$ ) was given to all patients, but not to control subjects, before imaging on both occasions.

A volume acquired inversion prepared fast spoiled gradient echo (FSPGR) of the spinal cord was acquired using phased array coils (TR $15.6 \mathrm{~ms}$, TE $4.2 \mathrm{~ms}$, TI $450 \mathrm{~ms}$, flip angle 20, matrix $256^{2}, 60 \times 1 \mathrm{~mm}$ sagittal slices). This was a 3D Fourier transform technique. Sagittal acquisition was chosen as it required fewer phase encodings in the slice select direction. A series of five contiguous $3 \mathrm{~mm}$ pseudoaxial slices were reformatted from the volume data set on the Signa using the centre of the C2/C3 intervertebral disc as a caudal landmark, with the slices perpendicular to the spinal cord. The images were then transferred to a Sun workstation (Sun Microsystems, Inc, Mountain View, CA, USA) and uniformity corrected. Using an automated program the images were then allocated random file names. A single observer, blinded to both to the patient details and the scan acquisition order, calculated the mean area of the slices using a technique described by Losseff $e t a l .{ }^{1}$

The spinal cord was also imaged with proton density and T2 weighted fast spin echo (FSE) images (TR $2500 \mathrm{~ms}$, effective TE 56/98 ms) with contiguous, $3 \mathrm{~mm}$ thick, sagittal slices using a phased array coil.

Axial dual echo FSE images of the brain (TR $3200 \mathrm{~ms}$, effective TE $15 / 90 \mathrm{~ms}$, field of view $24 \mathrm{~cm}$, matrix $256^{2}$, one excitation, slice thickness $3 \mathrm{~mm}$ ) were also acquired at baseline. The number of high signal T2 lesions, when present, was recorded. The volume of these lesions was calculated using a semiautomated, local thresholding technique

REPRODUCIBILITY

In five patients and five controls the spinal cord images were duplicated before the blinding process. After unblinding, the pairs of independently acquired spinal cord area measurements for these 10 patients were used to calculate the coefficient of variation for the technique. Error due to scan-rescan was not assessed in this study, but has been assessed previously. ${ }^{1}$ To ensure reproducibility over time, reference was made to a quality assurance protocol, which is ongoing in the department and involves serial scanning of control subjects. $^{8}$

STATISTICS

Changes of cord area within groups of patients were compared using a paired $t$ test. Compari- sons between patients and controls were performed using two-sample $t$ tests.

\section{Results}

The median age of the patients was 31 years (range 18-50 years). Twenty four were women. Baseline imaging was performed after a median of 5 weeks from the onset of symptoms (range 1-11 weeks) and imaging was repeated after a median of 12 months (range 11-17 months). Twenty nine patients presented with optic neuritis, nine with a brain stem syndrome and five with a spinal cord syndrome. One or more asymptomatic lesions (median 5, range 1-70) were identified as areas of high signal on T2 weighted brain MRI in $32(74 \%)$ of the patients. The median lesion volume was 0.4 $\mathrm{cm}^{3}$ (range $0-13.9 \mathrm{~cm}^{3}$ ). In two $(40 \%$ ) patients presenting with a spinal cord syndrome the symptomatic lesion was seen on spinal cord imaging. In one case the symptomatic lesion was in the cervical area and was associated with gadolinium enhancement. Fifteen healthy controls (eight women, seven men) with median age 29 years (range 23-49 years) were also studied on two occasions a median of 12 months (range 9-14 months) apart. The coefficient of variation for the measurement of spinal cord area using this technique was $1.4 \%$.

At baseline, the spinal cord area was significantly smaller in the subgroup of patients with an abnormal brain MRI than in controls (mean areas $73.9 \mathrm{~cm}^{3}$ and $78.1 \mathrm{~cm}^{3}$ respectively, $\mathrm{p}=0.03$ ) but not in the group as a whole (fig 1 ). Cord area in patients with normal brain MRI at presentation (mean area $76.5 \mathrm{~cm}^{3}$ ) did not differ significantly from the controls. The number and volume of cerebral lesions on T2 weighted imaging did not significantly correlate with spinal cord area.

When the five patients with spinal cord syndromes were excluded from the analysis, the difference in cord area between patients with brain lesions versus controls remained significant (mean $74.0 v 78.1 \mathrm{~mm}^{2}, \mathrm{p}=0.04$ ).

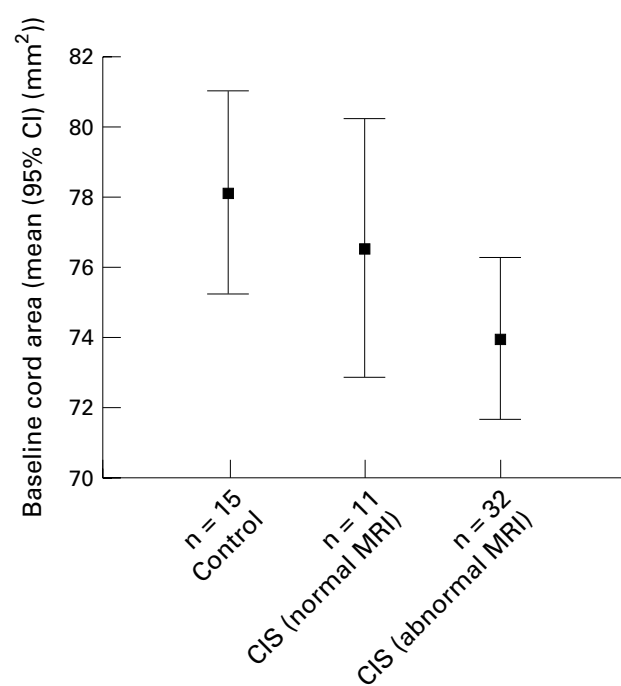

Figure 1 Spinal cord areas in controls and clinically isolated syndrome (CIS) subgroups with and without brain MRI abnormalities: means (95\% CI) on baseline scans. 
Table 1 Comparison of cord area and rate of atrophy in controls

\begin{tabular}{|c|c|c|c|c|c|}
\hline & \multirow[b]{2}{*}{ Control } & \multicolumn{4}{|c|}{ Clinically isolated syndrome } \\
\hline & & Total & No brain lesions & $\geqslant 1$ brain lesions & MS at follow up \\
\hline No of patients & 15 & 43 & 11 & 32 & 13 \\
\hline \multicolumn{6}{|l|}{ Age (y): } \\
\hline Mean & 31 & 31.4 & 32.8 & 30.9 & 33 \\
\hline Median & 29 & 31 & 31 & 31.5 & 31 \\
\hline Range & $23-49$ & $18-50$ & $25-50$ & $18-49$ & $19-49$ \\
\hline Sex & $7 \mathrm{M} / 8 \mathrm{~F}$ & $19 \mathrm{M} / 24 \mathrm{~F}$ & $7 \mathrm{M} / 4 \mathrm{~F}$ & $12 \mathrm{M} / 20 \mathrm{~F}$ & $4 \mathrm{M} / 9 \mathrm{~F}$ \\
\hline \multicolumn{6}{|c|}{ Baseline cord area $\left(\mathrm{mm}^{2}\right)$ : } \\
\hline Mean (SD) & $78.1(5.2)$ & $74.6(6.2)$ & $76.5(5.5)$ & $73.9(6.4)$ & $74.0(8.4)$ \\
\hline Range & $70.1-86.1$ & $63.2-91.1$ & $71.8-91.1$ & $63.2-90.2$ & $63.2-90.2$ \\
\hline & & 0.06 & 0.5 & 0.03 & 0.13 \\
\hline \multicolumn{6}{|c|}{ Follow up cord area $\left(\mathrm{mm}^{2}\right)$ : } \\
\hline Mean (SD) & $78.1(5.5)$ & $74.4(7.0)$ & $76.5(6.7)$ & $73.7(7.1)$ & $74.8(9.3)$ \\
\hline Range & $69.7-88.4$ & $62.8-92.8$ & $67.8-92.8$ & $62.8-90$ & $62.8-90$ \\
\hline${ }^{\star} \mathrm{p}$ from control & & 0.07 & 0.5 & 0.04 & 0.25 \\
\hline \multicolumn{6}{|l|}{ Atrophy/y $\left(\mathrm{mm}^{2}\right)$ : } \\
\hline Mean (SD) & $-0.1(2.3)$ & $-0.02(3.1)$ & $-0.05(3.5)$ & $-0.25(3.0)$ & $+0.8(3.6)$ \\
\hline Range & $-5.4-4.3$ & $-9.2-6.2$ & $-9.2-3.7$ & $-6.3-6.2$ & $-4.6-6.2$ \\
\hline tp within group & 0.99 & 0.67 & 0.98 & 0.63 & 0.45 \\
\hline${ }^{\star} \mathrm{p}$ from control & & 0.9 & 0.96 & 0.9 & 0.43 \\
\hline
\end{tabular}

`Two sample $t$ test (comparison with control).

†Paired sample $t$ test (comparison between time points within groups/subgroups).

Fourteen of 43 patients had one or more high signal lesions in the spinal cord on the sagittal FSE images. There was no difference in cord area between those patients with (mean $74.5 \mathrm{~mm}^{2}$ ) and those without (mean area 74.7 $\mathrm{mm}^{2}$ ) cord lesions.

The patient group with $\mathrm{T} 2$ brain lesions had a slightly higher proportion of women than the controls $(63 \% v 53 \%)$. This might raise the question whether sex differences in cord area itself had influenced the results. However, a previous evaluation of 15 males and 15 females using the same method, although showing a trend to smaller cord sizes in females, did not disclose a significant sex difference ${ }^{1}$. Furthermore, our patient group with normal brain MRI had an excess of men but did not exhibit a higher cord area than controls. We do not think that the minor sex differences in our subgroups could account for the results.

The practicalities of recruitment led to baseline scans being performed over a range of one to 11 weeks after the onset of the presenting syndrome. It is most unlikely that the baseline findings would have been different had they been obtained closer to the clinical episode, as measurable atrophy changes probably evolve over a longer period - the lack of change over the next year suggests that this was indeed the case.

After 1 year follow up, $13(30 \%)$ of the patients had had further clinical episodes leading to a diagnosis of clinically probable or definite multiple sclerosis. All of these patients had at least one high signal lesion on T2 weighted imaging of the brain. It was not possible to detect a significant change in the spinal cord area over the follow up period in either subgroup of patients with clinically isolated syndromes (with or without lesions on T2 weighted brain imaging) or in patients who developed clinical multiple sclerosis. Comparison of spinal cord area between the groups and subgroups had similar findings after 1 year (table 1).

\section{Discussion}

Cross sectional spinal cord area at the level of C2 was significantly smaller in the $74 \%$ of patients presenting with clinically isolated syndrome who had one or more T2 lesions on brain MRI than in a matched control group. This group of patients has been shown from previous long term follow up studies to have a high risk of developing multiple sclerosis ${ }^{7}$ and it is likely that in most cases we were studying multiple sclerosis at its earliest clinical stage. The percentage of patients in this study with high signal T2 brain lesions is comparable with that found in a larger study of patients with clinically isolated syndromes using modern MRI techniques, suggesting that this is a representative cohort. ${ }^{9}$ The baseline cord area in the patients who went on to develop multiple sclerosis within the year was also found to be reduced (mean area $74 \mathrm{~cm}^{3}$ ) compared with controls, but this did not achieve significance, perhaps due to the smaller sample size.

Patients presenting with a spinal cord syndrome made up $12 \%$ of the cohort. In only one of these patients was the symptomatic lesion seen in the cervical region in which the cord area was measured. Although this lesion enhanced after contrast administration, there did not seem to be swelling or focal atrophy associated with the lesion, and exclusion of this patient did not affect the results. The acquisition of spinal cord area measurements in images obtained after contrast administration in patients but not in controls, is a potential source of error in this study but it is thought to have been unlikely to effect the result. Notably, the signal contrast between the cord and the surrounding CSF, which is the critical feature enabling reproducible segmentation, seemed to be the same in both groups. This also ensured that blinding was preserved.

The rate of cord atrophy did not differ significantly from controls over the 1 year of follow up using the technique described, and in fact significant tissue loss over this period was not detected. This is likely due to the small 
amount of atrophy occurring over this short time period being counterbalanced by limitations in the sensitivity of the methods used. However, the reduction in cord area in these patients at presentation implies that atrophy has already begun to occur at and before this early clinical stage, at least in some patients. It is possible that the cervical cord measure of atrophy is particularly sensitive because of the length of the projection axons sampled.

A study of ventricular volume, a sensitive marker of change in brain volume, has also provided evidence that atrophy may occur early in multiple sclerosis. Significant increases in ventricular volume were found over 1 year in patients who had abnormal brain imaging at presentation with a clinically isolated syndrome and had a relapse leading to a diagnosis of multiple sclerosis, compared with those with normal baseline brain imaging and no further symptoms. ${ }^{10}$ In both the present study and the study of ventricular volume, the amount of atrophy detected was small. In addition, the finding of atrophy in the spinal cord arose only in a subgroup analysis of patients with clinically isolated syndromes and therefore this finding does need to be interpreted with some caution. Further studies are also needed to explore the relation between cord and brain atrophy in patients with clinically isolated syndromes.

Axonal loss may be a contributing cause to the cord atrophy found and has been found in postmortem studies to have occurred in this region of the spinal cord early in multiple sclerosis. ${ }^{6}$ However, although they both occur ${ }^{11}$, axonal loss and atrophy may not necessarily proceed together. Despite MR spectroscopy and magnetisation transfer imaging studies having found evidence compatible with focal tissue and axonal damage in the lesions of patients with clinically isolated syndromes, studies of normal appearing white matter of the brain suggest that widespread tissue and axonal damage is either mild or absent at this stage of the disease. ${ }^{12-15}$ Demyelination itself, with or without thin remyelinated fibres, should therefore also be considered a potential cause of such mild atrophy.

The small amount of atrophy found over the 1 year of this study contrasts with the rate of atrophy previously reported in established multiple sclerosis. ${ }^{2}$ Stevenson et al, using the same technique as us, detected a mean reduction in cord area of $2.98 \mathrm{~mm}^{2}$ in six patients with relapsing remitting multiple sclerosis in 1 year. The mean disease duration in these patients was 5.6 years (range 2-9 years).
Larger, serial MRI studies of patients with early relapsing remitting multiple sclerosis are required to determine when the rate of atrophy increases. The mechanisms of atrophy are also uncertain. For example, is it due to wallerian degeneration secondary to axonal transection in lesions or to pathological events occurring in the normal appearing tissues, which are independent of lesions? Understanding the mechanisms of atrophy will enable more rational targeting and timing of therapeutic interventions, which aim to prevent these widespread destructive changes. The use of serial multisequence MR investigations, which characterise different aspects of the evolving pathology, will be an important means of elucidating the basis of progressive atrophy.

PAB and JIO'R were sponsored by Schering AG. SML was sponsored by Biogen. The NMR Unit is supported by a generous grant from the multiple sclerosis Society of Great Britain and Northern Ireland. We thank Dr V L Stevenson and Mr M King for their assistance in this study.

1 Losseff NA, Webb SL, O'Riordan JI, et al. Spinal cord atrophy and disability in multiple sclerosis. A new reproducible phy and disability in multiple sclerosis. A new reproducible disease progression. Brain 1996;119:701-8.

2 Stevenson VL, Leary SM, Losseff NA, et al. Spinal cord atrophy and disability in MS: a longitudinal study. Neurology 1998;51:234-8.

3 Liu C, Edwards S, Gong Q, Roberts N. Three dimensional MRI estimates of brain and spinal cord atrophy in multiple sclerosis. F Neurol Neurosurg Psychiatry 1999;66:323-30.

4 Coles AJ, Wing MG, Molyneux P, et al. Monoclonal antibody treatment exposes three mechanisms underlying the clinical course of multiple sclerosis. Ann Neurol 1999;46:296-304.

5 Trapp BD, Peterson J, Ransohoff RM, et al. Axonal transection in the lesions of multiple sclerosis. N Engl f Med 1998; 338:278-85

6 Ganter P, Prince C, Esiri MM. Spinal cord axonal loss in multiple sclerosis: a post-mortem study. Neuropathol Appl Neurobiol 1999;25:459-67.

7 O'Riordan JI, Thompson AJ, Kingsley DP, et al. The prognostic value of brain MRI in clinically isolated syndromes of the CNS. A 10-year follow-up. Brain 1998;121:495-503.

8 Leary SM, Parker GJ, Stevenson VL, et al. Reproducibility of magnetic resonance imaging measurements of spinal cord atrophy: the role of quality assurance. Magn Reson Imaging 1999;17:773-6.

9 Brex PA, O'Riordan JI, Miszkiel KA, et al. Multisequence MRI in clinically isolated syndromes and the early development of MS. Neurology 1999;53:1184-90

10 Brex PA, Jenkins R, Fox NC, et al. Detection of ventricular enlargement in patients at the earliest clinical stage of multiple sclerosis. Neurology 2000;54:1689-91

11 Evangelou N, Esiri MM, Smith S, et al. Quantitative pathological evidence for axonal loss in normal appearing white matter in multiple sclerosis. Ann Neurol 2000;47:391-5.

12 Brex PA, Gomez-Anson B, Parker GJ, et al. Proton MR spectroscopy in clinically isolated syndromes suggestive of spectroscopy in clinically isolated syndromes sugge
multiple sclerosis. F Neurol Sci 1999;166:16-22.

13 Tourbah A, Stievenart JL, Abanou A, et al. Normalappearing white matter in optic neuritis and multiple appearing white matter in optic neuritis and multiple sclerosis: a comparative proto

14 Iannucci G, Tortorella C, Rovaris M, et al. Prognostic value of $M R$ and magnetization transfer imaging findings in patients with clinically isolated syndromes suggestive of multiple sclerosis at presentation. Am $\mathcal{f}$ Neuroradiol 2000;21:1034-8.

5 Kaiser JS, Grossman RI, Polansky M, et al. Magnetization transfer histogram analysis of monosymptomatic episodes of neurologic dysfunction: preliminary findings. $\mathrm{Am} \mathcal{f} \mathrm{Neu}$ roradiol 2000;21:1043-7. 